\title{
Bounds for Turbulent Transport
}

\author{
Peter Constantin \\ Department of Mathematics \\ The University of Chicago
}

November 17, 2018

We would like to pursue a mathematical approach to turbulence that is able to predict bulk average quantities of experimental and practical significance. Among such bulk average quantities, the Nusselt number $N$, the enhanced bulk average heat transfer due to convection, is perhaps the simplest physical objective of rigorous mathematical study of turbulence.

The question we wish to address here is: what are the optimal bounds on $N$ as a function of Rayleigh number $R$ ? What is the effect of the Prandtl number? Theoretical results ([1], [2], [3]) predict a maximal ultimate behavior of $N \sim \sqrt{R}$. The experimental (掼) findings indicate however that

$$
N \sim R^{q}
$$

where the reported values for $q$ belong approximately to the interval $\left[\frac{2}{7}, \frac{1}{3}\right]$ for large $R$. The exponents $2 / 7$ and $1 / 3$ have been discussed by several authors, ([5], [6]). The recent results of ([7]) favor an exponent of 3/10 with logarithmic corrections. We will describe below some very simple rigorous results.

The mathematical formulation of the problem is based on the three dimensional Boussinesq equations for Rayleigh-Bénard convection ([8]). These are a system of equations coupling the three dimensional Navier-Stokes equations

$$
\frac{\partial \mathbf{u}}{\partial t}+\mathbf{u} \cdot \nabla \mathbf{u}+\nabla p=\sigma \Delta \mathbf{u}+\sigma R T \mathbf{e}_{\mathbf{z}}, \quad \nabla \cdot \mathbf{u}=0
$$


to a heat advection-diffusion equation

$$
\frac{\partial T}{\partial t}+\mathbf{u} \cdot \nabla T=\Delta T
$$

The five unknowns, incompressible velocity, $\mathbf{u}=(u, v, w)$, pressure $p$, and temperature $T$ are functions of position $\mathbf{x}=(x, y, z)$ and time $t . R$ is the Rayleigh number and $\sigma$ is the Prandtl number. $\mathbf{e}_{\mathbf{z}}=(0,0,1)$ is the unit vector in the vertical direction. For simplicity of exposition the equations have been non-dimensionalized: the vertical variable $z$ is scaled so that it belongs to the interval $[0,1]$, the horizontal independent variables $(x, y)$ belong to a square $Q \subset \mathbf{R}^{\mathbf{2}}$ of side $L$. The boundary conditions are as follows: all functions $((u, v, w), p, T)$ are periodic in $x$ and $y$ with period $L ; u, v$, and $w$ vanish for $z=0,1$, and the temperature obeys $T=0$ at $z=1, T=1$ at $z=0$. We write

$$
\|f\|^{2}=\frac{1}{L^{2}} \int_{0}^{L} \int_{0}^{L} \int_{0}^{1}|f(x, y, z)|^{2} d x d y d z
$$

for functions and vectors $f$. We use $\langle\cdots\rangle$ for long time average:

$$
\langle f\rangle=\lim \sup _{t \rightarrow \infty} \frac{1}{t} \int_{0}^{t} f(s) d s .
$$

We write $\bar{f}$ the horizontal average

$$
\bar{f}=\frac{1}{L^{2}} \int_{0}^{L} \int_{0}^{L} f(x, y) d x d y .
$$

When a function depends on additional variables we write only the remaining variables after integration, so for instance

$$
\|\nabla \mathbf{u}(\cdot, t)\|^{2}=\frac{1}{L^{2}} \int_{0}^{L} \int_{0}^{L} \int_{0}^{1}|\nabla \mathbf{u}(x, y, z, t)|^{2} d x d y d z .
$$

The Nusselt number is given in terms of the long time average of the vertical heat flux:

$$
N=1+\left\langle\int_{0}^{1} b(z) d z\right\rangle
$$

with

$$
b(z, t)=\frac{1}{L^{2}} \int_{0}^{L} \int_{0}^{L} w(x, y, z, t) T(x, y, z, t) d x d y=\overline{w T}(z, t) .
$$


A consequence of the equations of motion are two additional formulas for the Nusselt number:

$$
\left\langle\|\nabla T\|^{2}\right\rangle=N
$$

and

$$
\left\langle\|\nabla \mathbf{u}\|^{2}\right\rangle=R(N-1) .
$$

The classical result of Howard, conditioned on on assumptions about statistical averages, is that $N$ is bounded at very large Rayleigh numbers by a multiple of $R^{\frac{1}{2}}$. The same kind of bound can be derived without any conditions ([9]). This bound is valid for all solutions, aspect ratios $L$ and Prandtl numbers $\sigma$ and is also valid in a rotating frame, at arbitrary rotation speed.

The system is not isotropic: the direction of gravity is singled out. Consider a function $\tau(z)$ that satisfies $\tau(0)=1, \tau(1)=0$, and express the temperature as

$$
T(x, y, z, t)=\tau(z)+\theta(x, y, z, t) .
$$

The role of $\tau$ is that of a convenient background ([10], [11], [12] ) that carries the inhomogeneous boundary conditions; thus $\theta$ obeys the same homogeneous boundary conditions as the velocity. Note that, because $\tau$ does not depend on $x, y$ one has

$$
T(x, y, z, t)-\bar{T}(z, t)=\theta(x, y, z, t)-\bar{\theta}(z, t) .
$$

The equation obeyed by $\theta$ is

$$
\left(\partial_{t}+u \cdot \nabla-\Delta\right) \theta=\tau^{\prime \prime}-w \tau^{\prime}
$$

where we used $\tau^{\prime}=\frac{d \tau}{d z}$. The horizontal average of the vertical velocity vanishes identically because of incompressibility

$$
\bar{w}(z, t)=0 .
$$

Therefore the quantity $b(z, t)$ can be written as

$b(z, t)=\frac{1}{L^{2}} \int_{0}^{L} \int_{0}^{L} w(x, y, z, t)(\theta(x, y, z, t)-\bar{\theta}(z, t)) d x d y=\overline{w(T-\bar{T})}(z, t)$ 
Multiplying the equation (9) by $\theta$ and integrating we obtain

$$
N+\left\langle\|\nabla \theta\|^{2}\right\rangle=2\left\langle-\int_{0}^{1} \tau^{\prime}(z) b(z) d z\right\rangle+\int_{0}^{1}\left(\tau^{\prime}(z)\right)^{2} d z .
$$

We will choose the background profile $\tau$ for simplicity to be a smooth profile concentrated in a boundary layer of width $\delta$,

$$
\tau(z)=P\left(\frac{z}{\delta}\right)
$$

with $P(0)=1$ and $P(s)=0$ for $s \geq 1$. Using only elementary facts (fundamental theorem of calculus, the boundary conditions and the Schwartz inequality) it is easy to see from (10) that

$$
|b(z, t)| \leq z\|\nabla \mathbf{u}(\cdot, t)\| \cdot\|\nabla(T-\bar{T})(\cdot, t)\|
$$

holds for any $z$. Let us define

$$
n=\left\langle\|\nabla(T-\bar{T})\|^{2}\right\rangle
$$

Note that, from the definition of $n$ and (5) it follows that

$$
n \leq N \text {. }
$$

From (12), (11) and (6) we obtain

$$
N \leq \frac{C}{\delta}+2 D \delta \sqrt{R(N-1)} \sqrt{n}
$$

with

$$
C=\int_{0}^{1}\left(\frac{d P(s)}{d s}\right)^{2} d s
$$

and

$$
D=\int_{0}^{1} s\left|\frac{d P(s)}{d s}\right| d s .
$$

Optimizing in $\delta<1$ we get

$$
\delta^{-1}=\sqrt{\frac{2 D}{C}}\{R(N-1) n\}^{\frac{1}{4}}+1
$$

and letting $P \rightarrow s$ we obtain

$$
N \leq 2(R n)^{\frac{1}{4}}(N-1)^{\frac{1}{4}}+1
$$

Thus we have proved 


\section{Theorem 1 Let}

$$
n=\left\langle\|\nabla(T-\bar{T})\|^{2}\right\rangle
$$

Then the Nusselt number (35, 5, (6) for three dimensional Rayleigh-Bénard convection satisfies

$$
N \leq 2^{\frac{4}{3}}(R n)^{\frac{1}{3}}+1 .
$$

If one has no additional information then, using $n \leq N$ in the inequality above we obtain the square-root bound

$$
N \leq 4 \sqrt{R}+1
$$

(The prefactor is not optimal. The search for optimal prefactors is better motivated for other systems, where the power law obtained rigorously coincides with the one observed in experiments. When that is the case then the rigorous results can match experiments with remarkable accuracy ([13])) . The exponent $1 / 3$ (or anything less than $1 / 2$ for that matter) have not been proven rigorously for the general system. The theorem above brings in the exponent $1 / 3$ in the general Boussinesq system conditionally, for slowly varying $n$. Another way by which the Nusselt number dependence on the Rayleigh number can be lower is if the Prandtl number is very high or in rotating (14]) convection. If the Prandtl number is infinity then the upper bound is closer to $1 / 3$. The equations of motion for infinite Prandtl number Rayleigh-Bénard convection ([15]) are

$$
-\Delta \mathbf{u}+\nabla p=R T e_{z}, \quad \nabla \cdot \mathbf{u}=0
$$

coupled with the advection-diffusion equation (21). Because (17) is time independent we say that $T$ obeys an active scalar equation.

An important observation, true even for the general case (11) is that, in view of the boundary conditions and incompressibility, not only the vertical component of velocity $w$ but also its normal derivative $\frac{\partial w}{\partial z}$ vanish at the vertical boundaries. Therefore we can write

$$
b(z, t)=\overline{\int_{0}^{z} d z_{1} \int_{0}^{z_{1}} \frac{\partial^{2} w}{\partial z^{2}}(\cdot, s, t) d s \int_{0}^{z}\left(\frac{\partial(T(\cdot, \sigma, t)-\bar{T}(\sigma, t))}{\partial z}\right) d \sigma .}
$$

Consequently

$$
|b(z, t)| \leq z^{\frac{5}{2}}\left\|\frac{\partial^{2} w(\cdot, t)}{\partial z^{2}}\right\|_{L^{\infty}}\|\nabla(T-\bar{T})(\cdot, t)\|
$$


where $\|f\|_{L^{\infty}}$ is the sup-norm. One can express $\frac{\partial^{2} w}{\partial z^{2}}$, in terms of $T-\bar{T}$. Indeed, eliminating the pressure from (17) one has

$$
\Delta^{2} w=-R \Delta_{h}(T-\bar{T})
$$

where $\Delta_{h}$ is the Laplacian in the horizontal directions $x$ and $y$. Using the boundary conditions we may write this as

$$
w=-R\left(\Delta_{D N}^{2}\right)^{-1} \Delta_{h}(T-\bar{T})
$$

where $\left(\Delta_{D N}^{2}\right)^{-1}$ is the inverse bilaplacian with homogeneous Dirichlet and Neumann boundary conditions. Taking two $z$ derivatives then yields

$$
\frac{\partial^{2} w}{\partial z^{2}}=-R B(T-\bar{T})
$$

where the linear operator $B$ is given by

$$
B=\frac{\partial^{2}}{\partial z^{2}}\left(\Delta_{D N}^{2}\right)^{-1} \Delta_{h}
$$

The temperature equation obeys a maximum principle so that

$$
0 \leq T \leq 1
$$

holds pointwise in space and time. Therefore

$$
0 \leq|T-\bar{T}| \leq 1
$$

holds. The operator $B$ is not bounded in $L^{\infty}$ but obeys a logarithmic extrapolation estimate. This means that higher derivatives enter logarithmically in the bound; the estimate

$$
\left\langle\|B(T-\bar{T})\|_{L^{\infty}}^{2}\right\rangle \leq C_{1}^{2}\left\{1+\log _{+} R\right\}^{4}
$$

follows from the bounds in ([16]). The constant $C_{1}$ can be computed explicitly. Therefore, using the same kind of background as above in (11) we obtain

$$
N \leq \int_{0}^{1}\left(\tau^{\prime}(z)\right)^{2} d z+2 \int_{0}^{1} z^{\frac{5}{2}}\left|\tau^{\prime}(z)\right|\left\langle\left\|\frac{\partial^{2} w(\cdot, t)}{\partial z^{2}}\right\|_{L^{\infty}}\|\nabla(T-\bar{T})\|\right\rangle d z
$$


and consequently

$$
N \leq \frac{C}{\delta}+2 E \delta^{\frac{5}{2}} C_{1} R\left\{1+\log _{+} R\right\}^{2} \sqrt{n}
$$

with $C$ defined in (14), $C_{1}$ coming from (24) and

$$
E=\int_{0}^{1} s^{\frac{5}{2}} P(s) d s .
$$

optimizing in $\delta<1$ we find

$$
\delta^{-1}=\left[\frac{5 E}{C} C_{1} R\left\{1+\log _{+} R\right\}^{2} \sqrt{n}\right]^{\frac{2}{7}}+1
$$

and then letting $P \rightarrow s$ we deduce

Theorem 2 There exists a constant $C_{2}$ such that the Nusselt number for the infinite Prandtl number equation is bounded by

$$
N \leq 1+C_{2} R^{\frac{2}{7}}\left\{1+\log _{+} R\right\}^{\frac{4}{7}} n^{\frac{1}{7}}
$$

If no additional information is given then, using $n \leq N$ in the theorem above we recover the result

$$
N \leq 1+C_{2}^{\frac{7}{6}} R^{\frac{1}{3}}\left\{1+\log _{+} R\right\}^{\frac{2}{3}}
$$

of ([16]). The theorem brings in the exponent $2 / 7$ as long as $n$ is not varying too much with $R$.

\section{Discussion}

There is no proof that the Nusselt number cannot ever scale like $R^{\frac{1}{2}}$ for exceedingly large $R$. The experimental data do not seem however to encounter this behavior. Mathematically, the Nusselt number represents the maximum (among all possible invariant measures) of the expected value of the diameter of the global attractor in the energy dissipation norm. The functions on the attractor are not arbitrary, and may have certain properties that explain the experimentally observed bounds $([1])$. In this paper we showed that if the ratio

$$
\frac{n}{N}=\frac{\left\langle\|\nabla(T-\bar{T})\|^{2}\right\rangle}{\left\langle\|\nabla T\|^{2}\right\rangle}
$$


is small then the Nusselt number dependence on Rayleigh is depleted.

Acknowledgment This work was partially supported by nsf-dms9802611 and by the ASCI Flash Center at the University of Chicago under DOE contract B341495.

\section{References}

[1] L.N. Howard, (1964), Heat transport in turbulent convection, J. Fluid Mechanics 17 405-432.

[2] L.N. Howard, (1964) Convection at high Rayleigh number, Applied Mechanics, Proc. 11th Cong. Applied Mech. (Ed. H. Grtler), pp. 1109-1115.

[3] R.H. Kraichnan, (1962), Turbulent thermal convection at arbitrary Prandtl number, Phys. Fluids 5, 1374-1389.

[4] F. Heslot, B. Castaing, and A. Libchaber, (1987), Transitions to turbulence in helium gas, Phys. Rev. A 36, 5870-5873 .

[5] B. Castaing, G. Gunaratne, F. Heslot, L. Kadanoff, A. Libchaber, S. Thomae, X.-Z. Wu, S. Zaleski and G. Zanetti, (1989), Scaling of hard thermal turbulence in Rayleigh-Benard convection, JFM 204, 1-30.

[6] B.I. Shraiman and E.D. Siggia (1990), , Heat transport in high-Rayleighnumber convection,, Phys. Rev. A 42, 3650-3653.

[7] J.J. Niemela, L. Skrbek, K.R. Sreenivasan, and R.J. Donnelly, (1999) "Turbulent convection at very high Rayleigh numbers", submitted to Nature.

[8] S. Chandrasekhar, (1961) Hydrodynamic and hydromagnetic stability, Oxford University Press, Oxford.

[9] C. R. Doering, P. Constantin, (1996), Variational bounds on energy dissipation in incompressible flows III. Convection, Phys. Rev E, 53 5957-5981. 
[10] C. R. Doering, P. Constantin, (1992), Energy dissipation in shear driven turbulence, Phys.Rev.Lett. 69, 1648-1651.

[11] P. Constantin, C. R. Doering, (1996), Heat transfer in convective turbulence, Nonlinearity 9, 1049-1060.

[12] P. Constantin, C. R. Doering, (1995), Variational bounds in dissipative systems, Physica D 82, 221-228.

[13] C. Doering, P. Constantin, (1998), Bounds for heat transport in a porous layer, J. Fluid Mechanics 376, 263-296.

[14] P. Constantin, C. Hallstrom, V. Putkaradze, (1999), Heat transport in rotating convection, Physica D 125, 275-284.

[15] S.-K. Chan, (1971), Infinite Prandtl Number Turbulent Convection, Stud. Appl. Math 50, 13-49.

[16] P. Constantin, C. R. Doering, (1999), Infinite Prandtl number convection, J. Stat. Phys., 94, 159-172. 